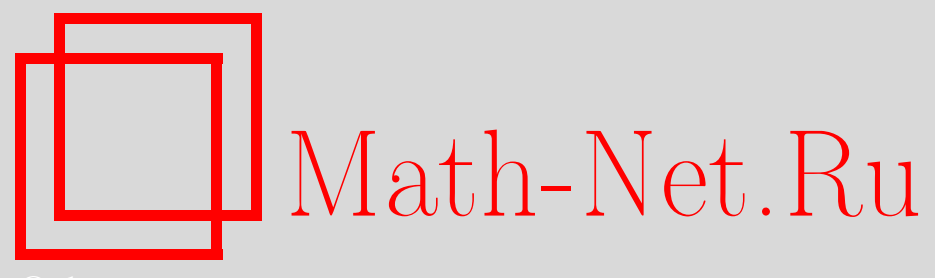

A. Оноприенко, Непрерывность дискретная и обычная, Квант, 2020, номер 2, 38-42

DOI: https://doi.org/10.4213/kvant20200203

Использование Общероссийского математического портала Math-Net.Ru подразумевает, что вы прочитали и согласны с пользовательским соглашением http://www . mathnet.ru/rus/agreement

Параметры загрузки:

IP: 54.224 .60 .19

26 апреля 2023 г., 16:18:08

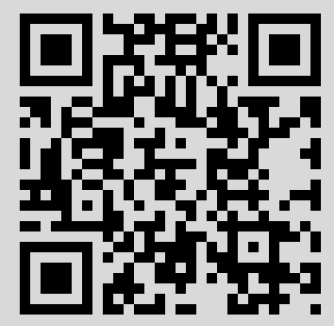




\section{Непрерывность дискретная и обычная}

\section{А.ОНОПРИЕНКО}

\section{Дискретная непрерывность}

Начнем с простой задачи.

Задача 1. В ряд выписано несколько чельх чисел, причем любые два соседних либо равны, либо отличаются на единицу. Первое число ряда равно -10, а последнее равно 10. Докажите, что в этой последовательности есть число 0.

Решение. По существу, эта задача говорит о следующем: начав с целого отрицательного числа и сдвигаясь на один, мы не можем попасть в положительное число, «перескочив» при этом ноль. Это утверждение выглядит совсем очевидно, но все же аккуратно докажем его. Найдем в нашем ряду первое неотрицательное число (по условию неотрицательные числа в ряду присутствуют, а значит, можно взять первое из них). Может ли оно быть больше нуля? Нет, так как в этом случае предыдущее число обязано было бы быть неотрицательным, но мы взяли первое неотрицательное число в этом ряду. Значит, первое неотрицательное число равно нулю.

Bonpoc. В каком месте предыдущего доказательства использовалось условие, что первое число ряда равно -10 ?

Похожим образом можно доказать более общее утверждение.

Статья написана по материалам математических кружков при МПГУ. Кружки открыты с 2016 года, а в 2018/19 и в 2019/20 учебных годах они получили поддержку Департамента образования города Москвы в рамках мероприятия «Детский университет МПГУ (организация работы кружков для московских школьников на базе МПГУ)».

DOI: https://doi.org/10.4213/kvant20200203
Дискретная теорема о промежуточном значении. Пусть имеется последовательность целых чисел, в которой любые два соседних числа или равны, или отличаются на единицу. Пусть а - первый элемент последовательности, $b$ - последний. Тогда для любого иелого числа с, лежащего между $a u b$, найдется элемент последовательности, равный с.

Приведем еще одну формулировку дискретной теоремы о промежуточном значении. Данная теорема в этой формулировке будет очень похожа на теорему о промежуточном значении для обычной непрерывности, которую мы обсудим ниже.

Заметим, что последовательность целых чисел $a_{1}, a_{2}, \ldots, a_{n}$ можно трактовать как функцию, отображающую отрезок $[1 ; n]$ натурального ряда в целые числа. Если записать эту последовательность как $a(1), a(2), \ldots$ $\ldots, a(n)$, можно увидеть полную аналогию с последовательностью значений функции $a$ в точках $1,2, \ldots, n$. Слова « $i$-й элемент последовательности» можно интерпретировать как «значение функции $a$ в точке $i$ ». (Правда, в данной терминологии функция $a$ определена лишь на натуральных числах, а не в любой действительной точке, как это бывает со многими функциями.) Можно обобщить это определение и считать, что функция $a$ определена не на числах $1,2, \ldots, n$, а на числах $k, k+1, \ldots, n$. В данном случае это соответствует последовательности $a_{k}, a_{k+1}, \ldots, a_{n}$ (или $a(k), a(k+1), \ldots, a(n)$, если записать по порядку значения, которые принимает функция $a$ в точках $k, k+1, \ldots, n)$.

Дискретная теорема о промежуточном значении (переформулировка). Пусть имеется функция $f$, определенная на натуральных числах $k, k+1, \ldots, n$ и принимающая иелье значения. Известно, что для любого $x=k, k+1, \ldots, n-1$ значения $f(x) u f(x+1)$ либо равны, либо отличаются на 1. Пусть $A=f(k), B=f(n)$. Тогда для любого иелого $C$, лежащего между $A$ и $B$, найдется такое иисло $x$, ито $f(x)=C$.

Разберем еще несколько задач, применяя дискретную теорему о промежуточном значении.

Задача 2. Первый тайм футбольного матча закончился со счетом 0:1, а второй 
тайм - со счетом 4:3. Докажите, что в некоторый момент счет на табло был ничейный.

Решение. Рассмотрим последовательность, элементами которой являются разности между мячами, забитыми первой и второй командами. Первый элемент последовательности представляет собой разность в начале второго тайма, последний элемент - разность в конце второго тайма. Вместе с каждым забитым мячом появляется новый элемент последовательности (т.е. каждый элемент последовательности соответствует обновлению счета на табло). Так как первый элемент последовательности равен -1, последний равен 1 и каждые два соседних значения отличаются на 1, по дискретной теореме о промежуточном значении есть элемент последовательности, равный нулю. Этот элемент соответствует моменту, когда счет на табло был ничейным.

Задача 3. В некоторой стране человек считается богатым, если его зарплата больше зарплать премьер-министра. В этой стране богатые мужчинь предпочитают жениться на бедных женшинах. Докажите, что можно установить премьер-министру такую зарплату, чтобы количество богатых мужчин было в точности равно количеству бедных женшин. (Все зарплаты в стране различные. Считайте, что зарплата бывает любым вещественным числом.)

Решение. Упорядочим по возрастанию все имеющиеся в стране зарплаты: $z_{1}, z_{2}, \ldots, z_{n}$, зарплату премьер-министра обозначим через $Z$. Определим последовательность натуральных чисел так: $a_{k}$ равно разности между количеством богатых мужчин и бедных женщин, если $z_{k}<Z<z_{k+1}$. Кроме того, $a_{0}$ (соответственно, $a_{n}$ ) равно той же разности, если $Z<z_{1}$ (соответственно, $Z>z_{n}$ ). При таком определении любые два соседних элемента последовательности $a_{k}$ и $a_{k+1}$ отличаются на 1, так как при соответствующем изменении зарплаты премьер-министра человек с зарплатой $z_{k+1}$ становится богатым, что уменьшает текущую разность на 1 , если этот человек был женщиной, или увеличивает на 1, если он был мужчиной. Первый элемент последовательности соответствует ситуации, когда все люди в стране богатые (кроме самого премьера), и тогда $a_{0}>0$. Соответственно, последний элемент после- довательности означает ситуацию, когда все люди бедные, и $a_{n}<0$. По дискретной теореме о промежуточном значении существует такое $k$, что $a_{k}=0$. Тогда установим зарплату премьер-министру, соответствующую этому значению $k$.

Задача 4. На окружности отмечены 77 точек, среди которых нет диаметрально противоположных. Докажите, ито можно провести диаметр через одну из этих точек так, что по обе стороны диаметра точек окажется поровну.

Решение. Проведем диаметр окружности через произвольную отмеченную точку и раскрасим одну из половинок круга в синий цвет, а другую - в красный. Если в половинках поровну точек, то нужный диаметр найден. В противном случае определим последовательность следующим образом: $a_{1}$ равно разности между количеством отмеченных точек в синей части и красной части для данного положения диаметра. Будем постепенно поворачивать диаметр вокруг центра окружности (раскрашенные половинки круга поворачиваются вместе с ним) и каждый раз, когда с диаметра уходит отмеченная точка либо попадает на него, мы записываем новый элемент последовательности (который тоже равен разности между количеством отмеченных точек в синей части и красной части). Последний элемент последовательности соответствует такому положению диаметра, когда он снова проходит через первую отмеченную точку. В этом положении диаметр поворачивается на $180^{\circ}$, а синяя и красная половинки меняются местами. Следовательно, последний элемент последовательности противоположен первому, т.е. один из них положителен, а второй отрицателен. Так как любые два соседних элемента последовательности отличаются на 1, по дискретной теореме о промежуточном значении найдется элемент последовательности, равный нулю. Осталось заметить, что этот элемент последовательности соответствует положению диаметра, проходящему через одну из отмеченных точек, так как общее количество точек нечетно.

Наконец, обсудим еще один тип дискретной непрерывности. Представим себе кузнечика, который прыгает по прямой вперед или назад. Прыжки кузнечика по длине не 
превосходят некоторого числа $C$. И если между точками старта и финиша пути имеется лужа длиной хотя бы $C$, то кузнечик обязательно попадет в лужу. Запишем это наблюдение в виде теоремы.

Дискретная теорема о промежуточном значении (еще одна переформулировка). Пусть имеется последовательность действительньх чисел $a_{1}, a_{2}, \ldots, a_{n}$, любые два соседних элемента которой отличаются не более чем на С. Кроме того, пусть х и $y$ - такие числа, ито $y-x \geq C$ и отрезок $[x ; y]$ содер жится в отрезке $\left[a_{1} ; a_{n}\right]$ (или $\left[a_{n} ; a_{1}\right]$, если $\left.a_{n}<a_{1}\right)$. Тогда существует элемент последовательности, лежащий в отрезке $[x ; y]$.

Этот вариант дискретной теоремы о промежуточном значении доказывается почти так же, как ее предыдущая версия: считая для определенности, что $a_{1} \leq x \leq a_{n}$, рассмотрите первое число последовательности, которое не меньше $x$.

Задача 5. Имеется 25 кусков сыра разного веса. Всегда ли можно один из этих кусков разрезать на две части и разложить сыр в два пакета так, что части разрезанного куска окажутся в разных пакетах, веса пакетов будут одинаковы и число кусков в пакетах также будет одинаково?

Решение. Покажем, что так всегда можно будет сделать. Отложим самый большой по весу кусок в сторону (пусть его вес равен $m_{25}$ ), а остальные 24 куска произвольно разложим в два пакета по 12 кусков. Отметим следующий факт: если суммарный вес сыра в первом пакете отличается от суммарного веса сыра во втором пакете менее чем на $m_{25}$, то можно разрезать отложенный кусок на две части и уравнять веса пакетов. Действительно, если первый пакет тяжелее второго на $\Delta m<m_{25}$, то разрежем отложенный кусок на части весом $\frac{m_{25}-\Delta m}{2}$ и $\frac{m_{25}+\Delta m}{2}$ и положим первую часть в первый пакет, а вторую - во второй.

Значит, осталось показать, что можно устроить так, чтобы веса первого и второго пакетов отличались менее чем на $m_{25}$ и при этом в каждом пакете было по 12 кусков. Пусть веса кусков в первом пакете равны $a_{1}, a_{2}, \ldots, a_{12}$, а во втором пакете $b_{1}, b_{2}, \ldots, b_{12}$. Если суммарные веса пакетов отличаются менее чем на $m_{25}$, то все доказа- но. Если же нет, то будем для определенности считать, что $a_{1}+a_{2}+\ldots+a_{12}+m_{25} \leq$ $\leq b_{1}+b_{2}+\ldots+b_{12}$. Организуем последовательное перекладывание: сначала обменяем местами куски $a_{1}$ и $b_{1}$, затем-куски $a_{2}$ и $b_{2}$ и т.д. Отметим, что так как отложенный кусок имеет максимальный вес среди всех, то после каждого перекладывания разность весов первого и второго пакетов меняется не более чем на $m_{25}$. А так как в начале процесса эта разность была не более $-m_{25}$, в конце процесса (когда мы поменяли местами все куски) - не менее $m_{25}$ и идем мы шагами не более $m_{25}$, то по второму варианту дискретной теоремы о промежуточном значении мы обязательно попадем в отрезок $\left[-\frac{m_{25}}{2} ; \frac{m_{25}}{2}\right]$. На этом шаге веса пакетов отличаются менее чем на $m_{25}$, и, как было показано выше, можно совершить требуемое разрезание отложенного куска.

\section{Обычная непрерывность}

Возьмем карандаш и начнем рисовать, не отрывая его от бумаги. В результате мы получим некоторую непрерывную линию. Многие функции, которые изучаются в школе, имеют график, который можно нарисовать, не отрывая карандаш от бумаги: например, $y=x^{3}, y=\sqrt{x}$ и другие. График такой функции (называемой непрерывной) выглядит как линия, которая не имеет разрывов. (Отметим, что мы не даем точное определение непрерывной функции - ведь для этого требуется объяснить, что такое линия, не имеющая разрывов! На самом деле в математике определение линии, которую можно нарисовать, не отрывая карандаш от бумаги, приводится через определение непрерывной функции, а не наоборот.) Функция $y=\frac{1}{x}$, определенная во всех точках, кроме 0, имеет две ветви, каждую из которых можно нарисовать, не отрывая карандаш от бумаги, и она тоже непрерывна на своей области определения. В отличие от функций из предыдущего раздела эти функции определены на бесконечных множествах, и поэтому дискретная теорема о промежуточном значении для них не подходит. Рассмотрим следующее «определение» непрерывной функции, 
которое удобно использовать в задачах. Интуитивно оно означает, что функция не совершает резких скачков и изменяется плавно.

«Определение». Функция $y=f(x)$ непрерывна на своей области определения, если при малых изменениях значения аргумента мало меняется значение функции.

Почему слово «определение» взято в кавычки? Дело в том, что написанный выше текст не является определением в строгом смысле. В самом деле, это значит «мало меняется»? Однако этому «определению» можно придать точный смысл, что делают в университетском курсе математического анализа. В этом же курсе доказывают следующую теорему о промежуточном значении. Ее формулировка очень похожа на дискретную теорему о промежуточном значении, но ее доказательство значительно сложнее и потому остается за рамками статьи.

Теорема о промежуточном значении. Пусть числовая функиия $f$ определена на отрезке $[a ; b]$ и непрерывна на этом отрез-

ке. Пусть $f(a)=A, \quad f(b)=B$. Тогда для любого числа $C$, лежащего между $A$ u $B$, найдется такое число $x$, ито $f(x)=C$.

Замечание. Приведенное выше «определение» непрерывной функции можно вполне строго применить к функциям, которые мы вводили для переформулировки дискретной теоремы о промежуточном значении. Для этого под словом «мало» нужно понимать «не более чем на единицу».

Задача 6. В противоположных углах квадратного пруда со стороной 100 метров сидели два гуся. Поплавав по пруду, они оказались в двух соседних углах. Докажите, что в некоторый момент времени расстояние между кончиками их клювов было равно 110 метрам.

Решение. Пусть гуси плавали от момента времени $t_{1}$ до момента времени $t_{2}$. Рассмотрим функцию $f$, ставящую в соответствие моменту времени $t$ расстояние между кончиками клювов гусей в этот момент. Эта функция будет непрерывной: гуси плавают без резких скачков, и поэтому за малое изменение времени расстояние между ними поменяется на малую величину. Так как $f\left(t_{2}\right)=$
$=100 \sqrt{2}<110$, a $f\left(t_{2}\right)=100<110$, то по теореме о промежуточном значении найдется такой момент $t$, что $f(t)=110$.

Задача 7. Докажите, что любой выпуклый многоугольник можно одним прямолинейным разрезом разбить на два равновеликих многоугольника.

Решение. Поместим многоугольник между двумя параллельными прямыми $a$ и $b$, не пересекающими этот многоугольник. Пусть $d$ - расстояние между этими прямыми. Для каждого $x \in[0 ; d]$ можно провести прямую $c_{x}$, параллельную $a$ и $b$, лежащую между этими прямыми и расстояние от которой до $a$ будет равно $x$. Рассмотрим функцию $f$, которая по числу $x$ определяет площадь части многоугольника, лежащую между прямыми $a$ и $c_{x}$. Эта функция непрерывна: при малых изменениях $x$ прямая $c_{x}$ будет сдвигаться на небольшое расстояние, и площадь части многоугольника между $a$ и $c_{x}$ тоже мало изменится. Так как $f(0)=0, f(d)=S-$ площади многоугольника, найдется такое $x=x_{0}$, что $f\left(x_{0}\right)=\frac{S}{2}$. Тогда прямая $c_{x_{0}}$ будет искомой.

Задача 8. В противоположных концах диаметра $A B$ окружности сидят два таракана. По команде они побежали по окружности в одном направлении (например, против часовой стрелки) и через минуту поменялись местами (движение тараканов не обязательно равномерное). Докажите, ито в какой-то момент соединяющая их хорда была перпендикулярна диаметру $A B$.

Решение. Пусть тараканы бежали от момента времени $t_{1}$ до $t_{2}$. Для всех моментов времени $t$ между ними (не включая $t_{1}$ и $t_{2}$ ) определим следующую функцию $f$. Пусть $C_{t}, D_{t}-$ положения первого и второго таракана соответственно, $O_{t}$ - точка пересечения $A B$ и $C_{t} D_{t}$. Значение функции $f(t)$ равно градусной мере угла $A O C_{t}$. В моменты времени $t$, близкие к $t_{1}$, значение функции $f(t)$ близко к $0^{\circ}$. А в моменты времени $t$, близкие к $t_{2}$, значение функции $f(t)$ близко к $180^{\circ}$. Кроме того, функция $f$ непрерывна, поскольку положение точек $C_{t}, D_{t}$ и $O_{t}$ меняется непрерывно. А значит, согласно теореме о промежуточном значении, существует момент времени $t$, когда $f(t)=90^{\circ}$.

Bопрос. Почему функция $f$ не определена в точках $t_{1}$ и $t_{2}$ ? 


\section{Задачи для самостоятельного решения}

9. В ряд стоят 20 сапог: 10 правых и 10 левых. Докажите, что найдутся 10 сапог, стоящих подряд, среди которых поровну правых и левых.

10. Грани восьми единичных кубиков окрашены в черный и белый цвета так, что черных и белых граней поровну. Докажите, что из этих кубиков можно сложить куб $2 \times 2 \times 2$, на поверхности которого черных и белых квадратиков поровну.

11. В некоторых клетках квадрата $50 \times 50$ стоят числа 1 и -1 , причем сумма всех чисел не больше 100 и не меньше -100. Докажите, что есть квадрат $25 \times 25$, абсолютная величина суммы чисел в котором не превосходит 25.

12. Дан выпуклый многоугольник и точка а) вне, б) внутри него. Докажите, что его можно разбить на две равновеликие части прямой, проходящей через заданную точку.

13. Пусть $x_{1}, x_{2}, \ldots, x_{n}$ - некоторые числа, принадлежащие отрезку [0;1]. Докажите, что на этом отрезке найдется такое число $x$, что

$$
\frac{1}{n}\left(\left|x-x_{1}\right|+\left|x-x_{2}\right|+\ldots+\left|x-x_{n}\right|\right)=\frac{1}{2} \text {. }
$$

\section{Список литературы для дальнейшего изучения}

1. С.Табачников. Соображения непрерывности. - «Квант», 1987, №9.

2. А.Д.Блинков, В.М.Гуровии. Непрерывность. - М.: МЦНМО, 2015.

3. А.В.Шаповалов. Принцип узких мест. - М.: МЦНМО, 2008.

\section{Вокругточки на медиане}

\section{Д.ПРОКОПЕНКО, Д.ШВЕЦОВ}

В этой статье нас ждет обзорная экскурсия от древнегреческих шедевров геометрии до задач современных олимпиад. Мы постараемся проследить, как непростые задачи наших дней перекликаются с находками древних.

На заочном туре IX Олимпиады по геометрии имени И.Ф.Шарыгина была предложена такая задача (автор - Ф.Ивлев).

Задача 1. Вписанная в треугольник $A B C$ окружность касается сторон BC, $C A, A B$ в точках $A_{1}, B_{1}, C_{1}$ соответственно. Перпен-

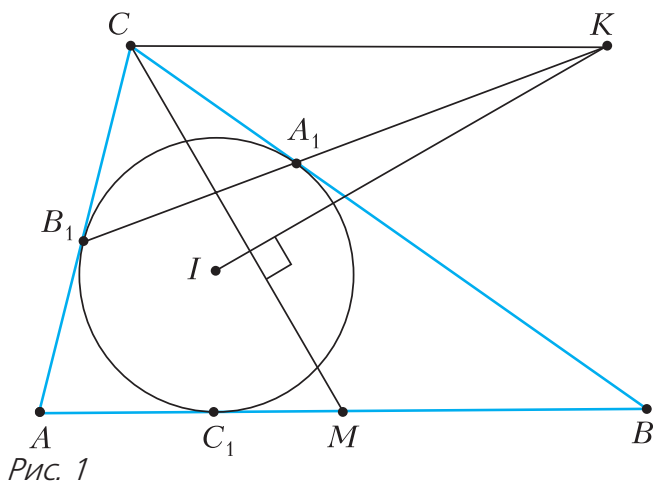

DOI: https://doi.org/10.4213/kvant20200204 дикуляр из центра I этой окружности на медиану CM пересекает прямую $A_{1} B_{1}$ в точке K (рис.1). Докажите, что $C K \| A B$.

Несмотря на простую и интересная формулировку, так сразу и не ясно, как к задаче подступиться. Решение же из книги [1] использует полярное преобразование, методы проективной геометрии, понятие бесконечно удаленной точки - требуется эрудиция. Неужели к задаче с таким элегантным условием нет более простого подхода? Есть! Оказывается, эта задача является вариацией следуещего классического факта [2]:

Теорема 1. Вписанная в треугольник $A B C$ окружность касается сторон $B C, C A, A B$ в точках $A_{1}, B_{1}, C_{1}$ соответственно. Пусть прямая $C_{1} I$ пересекает прямую $A_{1} B_{1}$ в точке $P$ (рис.2). Тогда прямая СР содержит медиану треугольника $A B C$.

Давайте сначала с помощью этой теоремы решим задачу 1 , а затем докажем и саму теорему.

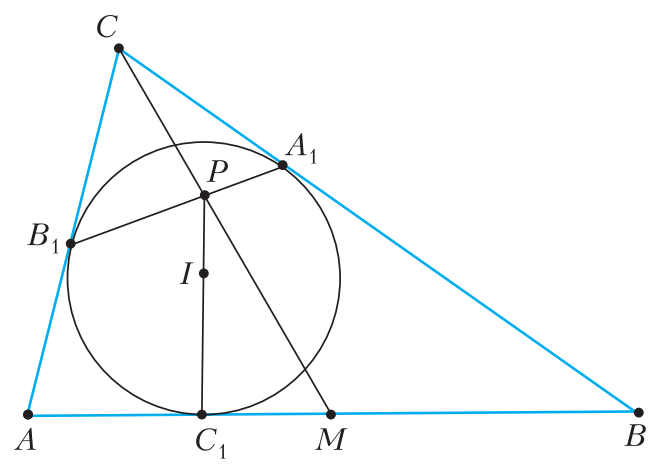

Puc. 2 